\title{
Black holes from nucleating strings
}

\author{
Jaume Garriga \\ Tufts Institute of Cosmology, Department of Physics and Astronomy, Tufts University, Medford, Massachusetts 02155 \\ and Institute for Theoretical Physics, University of California, Santa Barbara, California 93106-4030 \\ Alexander Vilenkin \\ Tufts Institute of Cosmology, Department of Physics and Astronomy, Tufts University, Medford, Massachusetts 02155 \\ and Lyman Laboratory of Physics, Harvard University, Cambridge, Massachusetts 02138
}

(Received 12 June 1992; revised manuscript received 9 December 1992)

\begin{abstract}
We evaluate the probability that a loop of string that has spontaneously nucleated during inflation will form a black hole upon collapse, after the end of inflation. We then use the observational bounds on the density of primordial black holes to put constraints on the parameters of the model. Combining these constraints with current upper limits on the expansion rate during inflation, we conclude that the density of black holes formed from nucleating strings is too low to be observed. Also, constraints on domain wall nucleation and monopole pair production during inflation are briefly discussed.
\end{abstract}

PACS number(s): 98.80.Cq, 03.70.+k, 97.60.Lf

\section{INTRODUCTION}

It has been proposed $[1,2]$ that loops of string can spontaneously nucleate during an inflationary period of expansion in the early Universe. This is a quantum tunneling process, somewhat analogous to the spontaneous nucleation of spherical bubbles of true vacuum in the problem of false vacuum decay [3]. It has been shown that loops can nucleate at considerable rates provided that their tension $\mu$ is not much larger than $H^{2}$ (here $H$ is the expansion rate during inflation) and, in this case, the distribution of loops emerging from inflation may in principle have significant cosmological consequences.

In this scenario, loops nucleate with sizes of the order of $H^{-1}$, and are subsequently stretched to large sizes by the inflationary expansion. After inflation, in the radiationdominated era, the loops eventually fall within the Hubble radius and start oscillating under their tension.

Because the instanton describing the nucleation of a loop is maximally symmetric, the nucleated loops tend to be nearly circular. It is well known that an exactly circular loop would form a black hole upon collapse [4-6]. However, the nucleated loops will not be exactly circular. The reason is that, during inflation, quantum fluctuations on the string on subhorizon scales generate shape instabilities as their wavelengths become larger than the horizon [7, 8] (see also [9]). The question then arises of how many of the nucleated loops will be circular enough to form a black hole after the end of inflation. This is the problem we address in the present paper.

The plan of the paper is the following. In Sec. II we calculate the probability of black hole formation from a nucleating string. In Sec. III we use this result and the observational bounds on the abundance of primordial black holes to put a constraint on the parameters of the model (which are essentially $\mu$ and $H$ ). An additional constraint from the emission of gravitational waves by the strings is also considered. In Sec. IV, we briefly discuss observational constraints on domain wall nucleation and monopole-antimonopole pair production, which can also occur during inflation [1]. We summarize our conclusions in Sec. V. Some details are left to the appendices. Appendix A deals with linearized perturbations on a circular loop of string in flat space, and in Appendix B we consider the effect of damping processes on the probability of black hole formation.

\section{PROBABILITY OF BLACK HOLE FORMATION}

In this section we estimate the probability that a loop of string that has nucleated during inflation will form a black hole once it collapses under its tension, after the end of inflation.

The classical world sheet of a circular loop of string that has nucleated during inflation is given by [1]

$$
R(t)=H^{-1} \sqrt{e^{2 H\left(t-t_{0}\right)}+1},
$$

where $R$ is the physical radius of the loop, $H$ is the expansion rate of the inflationary Universe, and $t$ is the usual cosmological time in the flat Friedmann-RobertsonWalker coordinates. The parameter $t_{0}$ can be interpreted (in a loose sense) as the time at which the loop nucleates. Throughout this paper (unless otherwise stated) we shall work in the approximation in which the strings are infinitely thin, although nucleation of strings whose thickness is not much smaller than $H^{-1}$ is also possible [2].

The loops nucleate with a size of the order of the horizon size $R \sim H^{-1}$, and afterwards they are stretched by the inflationary expansion. For $R \gg H^{-1}$ they grow proportionally to the scale factor

$$
R(t) \approx H^{-1} e^{H\left(t-t_{0}\right)} .
$$


After inflation, in the radiation-dominated era, the loops continue to be stretched by the expansion of the Universe, $R(t) \propto t^{1 / 2}$, until they enter the cosmological horizon, at $t \sim R$. Soon after the loop comes within the horizon, the effects of expansion become negligible and the loop starts behaving approximately as it would behave in flat space.

Let $R_{c}$ be the radius of a particular loop at horizon crossing. The mass of this loop is $2 \pi R_{c} \mu$, where $\mu$ is the string tension. The Schwarzschild radius corresponding to this mass is

$$
R_{S}=4 \pi G \mu R_{c} .
$$

As the loop shrinks under its tension, its rest mass is converted into kinetic energy, so that the total energy of the loop remains constant. It is clear that if the loops were exactly circular, they would all eventually shrink to a size smaller than $R_{S}$, thus forming black holes (of course this argument assumes that $R_{S}$ is larger than the thickness of the string, which will always be true for sufficiently large loops).

However, loops nucleated during inflation will not be exactly circular. Quantum fluctuations around the symmetric solution (1) will cause small departures from the circular shape, and this will determine the probability of black hole formation. Consider a circular loop of radius $R(t)$ lying in the $z=0$ plane and centered at the origin. Using cylindrical coordinates $(\rho, \theta, z)$, we can parametrize a perturbed (noncircular) loop as follows:

$$
\begin{aligned}
& \rho(\theta, t)=R(t)+\Delta_{r}(\theta, t), \\
& z(\theta, t)=\Delta_{t}(\theta, t) .
\end{aligned}
$$

That is, we decompose the perturbations into a radial part $\Delta_{r}$ parallel to the plane of the unperturbed loop, and a transverse part $\Delta_{t}$ perpendicular to that plane. In Eq. (3), $\rho$ and $z$ are physical coordinates (rather than comoving).

To study the evolution of a loop, we shall divide its history into three epochs: (a) The loop nucleates and then expands during inflation; (b) after inflation, while the loop is larger than the cosmological horizon it is stretched by the expansion until it crosses the horizon; (c) once the loop comes within the horizon, it shrinks under its tension. We will follow the evolution of the perturbations $\Delta_{r}, \Delta_{t}$ through the epochs (a), (b), and (c). For this it will be useful to expand them in Fourier modes:

$$
\Delta_{r}=\sum_{L=2}^{\infty}\left[\Delta_{r, L}^{(1)} \frac{\cos L \theta}{\sqrt{\pi}}+\Delta_{r, L}^{(2)} \frac{\sin L \theta}{\sqrt{\pi}}\right],
$$

and similarly for $\Delta_{t}$. Note that the sum does not include the modes with $L=0$ and $L=1$. This is because, as it was shown in Ref. [8], such modes do not correspond to true perturbations, but only to spatial rotations and spacetime translations of the unperturbed solution (1).

(a) Evolution during inflation. In the process of nucleation, the perturbations $\Delta_{\lambda, L}^{(i)}(i=1,2 ; \lambda=t, r)$ have to be treated as quantum variables. The linearized theory of quantum fluctuations around the classical solution (1) was studied in Ref. [8], using a covariant formalism. The world sheet (1) has the internal geometry of a (1+1)dimensional de Sitter space [1], and it was shown that the perturbations $\Delta_{r}$ and $\Delta_{t}$ behave as two uncoupled scalar fields of tachyonic mass $m^{2}=-2 H^{2}$ "living" in this lower-dimensional de Sitter space. The symmetries of the problem suggest that when the string nucleates, the fields $\Delta_{r}, \Delta_{t}$ should be in a de Sitter-invariant quantum state (see also Ref. [10]). This state was constructed in Ref. [8] using the Heisenberg picture. The corresponding Schrödinger picture wave functional $\Psi\left(\left\{\Delta_{\lambda, L}^{(i)}\right\}\right)$ can be obtained using well-known manipulations (see, e.g., [10]). Since we are dealing with a linearized theory, the probability distribution associated with $\Psi$ has the Gaussian form

$$
\mathcal{P}\left(\left\{\Delta_{\lambda, L}^{(i)}\right\}\right)=|\Psi|^{2}=\prod_{\lambda, i, L}\left(2 \pi \sigma_{\lambda, L}^{2}\right)^{-1 / 2} \exp \left[\frac{-\left(\Delta_{\lambda, L}^{(i)}\right)^{2}}{2 \sigma_{\lambda, L}^{2}}\right],
$$

where the standard deviations $\sigma_{\lambda, L}$ are given by [8]

$$
\sigma_{\lambda, L}^{2}(t) \equiv\left\langle\Psi\left|\left(\Delta_{\lambda, L}^{(i)}\right)^{2}\right| \Psi\right\rangle=\frac{1}{2 \mu L}\left[\frac{H^{2} R^{2}(t)}{L^{2}-1}+1\right] .
$$

For wavelengths much larger than the horizon, $H R \gg 1$, we have $\sigma_{L} \propto R$, i.e., the amplitude of the perturbations is conformally stretched by the expansion (recall that $R$ grows like the scale factor during inflation). For wavelengths much smaller than the horizon the second term in (6) dominates and we have $\sigma_{L} \approx$ const, as expected. This second term is just the flat space contribution to the quantum fluctuations, and we shall subtract it in what follows in order to avoid the well-known ultraviolet divergences in $\left\langle\Delta_{\lambda}^{2}\right\rangle$.

Soon after a given wavelength becomes larger than the horizon, it can be treated as classical. Here we adopt the point of view that Eq. (5) gives the probability distribution for the initial amplitudes of the perturbations, which will evolve classically thereafter.

(b) After inflation: while the loop is larger than the cosmological horizon. As we mentioned before, the loops are conformally stretched until they enter the horizon. Since we do not have analytical solutions to describe this epoch, we shall use the following approximations $[11,12]$ : for wavelengths larger than the horizon the amplitude of the perturbations is conformally stretched by the expansion, whereas for wavelengths within the horizon the amplitude of the perturbations remains constant.

Perturbations with wave number $L$ enter the horizon at time $t_{L} \sim R\left(t_{L}\right) / L$. At this time we have $\sigma_{L}=\left[2 \mu L\left(L^{2}-\right.\right.$ $1)]^{-1 / 2} H R\left(t_{L}\right)$ (as mentioned before, we subtract the flat space contribution to $\sigma_{L}$ ). The loop enters the horizon at the time

$$
t_{c} \sim R\left(t_{c}\right) \sim \frac{R^{2}\left(t_{L}\right)}{t_{L}} \sim L R\left(t_{L}\right) .
$$

Since the amplitude of the perturbations is frozen after $t_{l}$, we also have 


$$
\begin{aligned}
\sigma_{L}\left(t_{c}\right) & =\left[2 \mu L\left(L^{2}-1\right)\right]^{-1 / 2} H R\left(t_{L}\right) \\
& =\left[2 \mu L^{3}\left(L^{2}-1\right)\right]^{-1 / 2} H R_{c}
\end{aligned}
$$

at the time $t_{c}$.

(c) Loops inside the horizon. Once a loop comes within the horizon we can ignore the effects of the expansion of the Universe and consider the collapse of a loop in flat space. In this case the evolution of the perturbations can be solved analytically. The details can be found in Appendix A. We find that during this period the behavior of radial perturbations $\Delta_{r}$ is different from that of transverse perturbations $\Delta_{t}$. The amplitude of radial perturbations shrinks by a factor of $L$ as the loop collapses, while the amplitude of transverse perturbations remains constant. For $R \ll R_{c}$ we find

$$
\begin{gathered}
\sigma_{r, L} \approx\left(2 \mu L^{7}\right)^{-1 / 2} H R_{c}, \\
\sigma_{t, L} \approx\left(2 \mu L^{5}\right)^{-1 / 2} H R_{c} .
\end{gathered}
$$

Equation (8), together with (5), is the first important result of this paper. It allows us to assign probabilities to different string configurations (parametrized by $\Delta_{\lambda, L}^{(i)}$ ) at the time when the radius of the unperturbed loop enters the Schwarzschild radius $R_{S}$.

The dynamics of gravitational collapse and black hole formation from a given loop configuration is, of course, a complicated issue that cannot be treated in detail analytically. However, we know that if the amplitude of the perturbations is much smaller than $R_{S}$ at the time $t_{S}$ when the unperturbed loop enters the Schwarzschild radius, then a black hole will form. On the contrary, if the perturbations are large compared with $R_{S}$ the loop will probably self-intersect and fragment into smaller loops before it shrinks to a size $R_{S}$ (the resulting loops will be far from circular, so a black hole will never form in this case). To compute the probability of black hole formation, we shall count a particular loop as a black hole with Gaussian weight:

$$
e^{-\delta^{2} / \alpha^{2} R_{S}^{2}}
$$

Here $\delta$ is the rms perturbation when one averages over the circumference of the loop (at time $t_{S}$ ):

$$
\delta^{2} \equiv \frac{1}{2 \pi} \int_{0}^{2 \pi} \Delta^{2}\left(\theta, t_{S}\right)=\frac{1}{2 \pi} \sum_{i, \lambda, L}\left(\Delta_{\lambda, L}^{(i)}\right)^{2},
$$

where we have used (4). In Eq. (9), $\alpha$ is an unknown parameter of order 1 , which models our lack of knowledge on the dynamical details. We should emphasize that the precise form of the "Gaussian window" (9) is not very important here; we have taken the Gaussian shape just for computational convenience. Had we taken, for instance, a step function window, our final conclusions would be the same.

To find the probability of black hole formation from a loop that has nucleated during inflation, we integrate over all possible loop configurations with measure given by the probability distribution (5)-(8), times the window function (9):

$$
\begin{aligned}
\mathcal{P}_{\mathrm{BH}} & =\int|\Psi|^{2} e^{-\delta^{2} /\left(\alpha R_{S}\right)^{2}} \prod_{\lambda, i, L} d \Delta_{\lambda, L}^{(i)} \\
& =\prod_{\lambda=r, t} \prod_{L=2}^{\infty}\left(1+\frac{\sigma_{\lambda, L}^{2}}{\pi \alpha^{2} R_{S}^{2}}\right)^{-1} .
\end{aligned}
$$

Using (8), we can rewrite it as

$$
\mathcal{P}_{\mathrm{BH}}\left(L_{*}\right)=\prod_{L=2}^{\infty}\left(1+2 \frac{L_{*}^{5}}{L^{5}}\right)^{-1}\left(1+2 \frac{L_{*}^{5}}{L^{7}}\right)^{-1},
$$

where we have introduced the notation

$$
L_{*} \equiv\left[16 \pi^{2} B(\alpha G \mu)^{2}\right]^{-1 / 5} \text {. }
$$

Here $B \equiv 4 \pi \mu / H^{2}$ is the Euclidean action of the instanton describing the nucleation of the loop. Equation (11) is the main result of this section. It allows us to find the probability of black hole formation as a function of the parameters of the model $\left(\mu\right.$ and $H$ ). Note that $\mathcal{P}_{\mathrm{BH}}$ does not depend on $R_{c}$, the radius of the loop at horizon crossing. Note, also, that the dependence on the parameters $H, \mu$, and $\alpha$ is only through the combination $L_{*}$.

The physical meaning of $L_{*}$ is the following. Modes with $L \gg L_{*}$ have $\sigma_{L} \ll \alpha R_{S}$. These modes will always have amplitudes much smaller than the Schwarzschild radius and they will not contribute to $\mathcal{P}_{\mathrm{BH}}$. This is also clear from (11). Therefore $L_{*}$ is basically the number of modes whose amplitude can be large enough to prevent the formation of a black hole.

The function $\mathcal{P}_{\mathrm{BH}}\left(L_{*}\right)$ cannot be given in closed form. However, one can compute it numerically to arbitrary precision by including a sufficient number of terms in the product (11). A plot of $\mathcal{P}_{\mathrm{BH}}$ versus $L_{*}$ is given in Fig. 1. From the graph we see that the probability decays (essentially) as an exponential function of $L_{*}$.

In deriving Eq. (11) we have neglected damping processes, such as gravitational radiation and friction. In

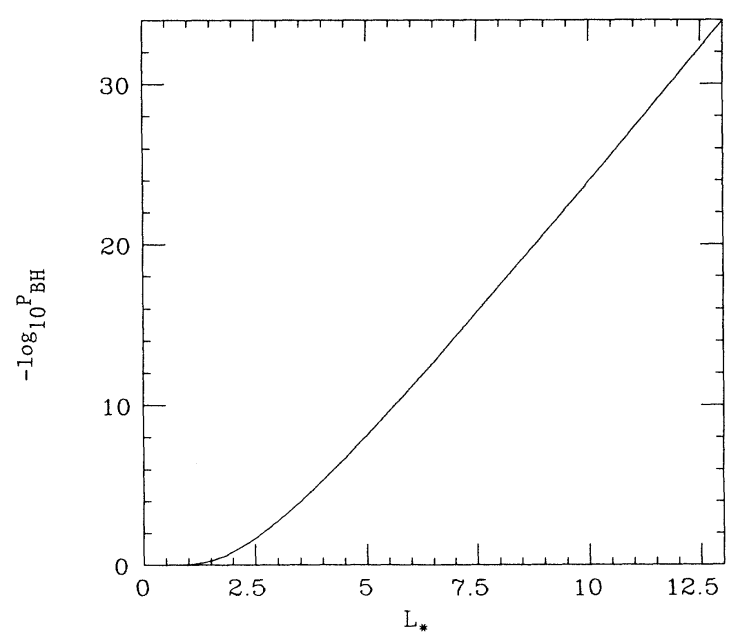

FIG. 1. The function $\mathcal{P}_{\mathrm{BH}}\left(L_{*}\right)$ can be computed numerically from Eq. (11) to arbitrary precision. We see that, essentially, the projability of black hole formation decays as an exponential function of $L_{*}$. 
principle these could reduce the amplitude of the perturbations and therefore increase the probability of black hole formation. In Appendix B we briefly discuss the damping mechanisms and the limits in which they can be neglected. We find that damping can be safely ignored from the calculation of $\mathcal{P}_{\mathrm{BH}}$ provided that $G \mu$ is in the range

$$
\left(\alpha^{2} B\right)^{-2 / 9}\left(\frac{m_{P}}{M}\right)^{5 / 9} \lesssim G \mu<5 \times 10^{-2}\left(\alpha^{2} B\right)^{1 / 3} \text {, }
$$

where $m_{P}$ is the Planck mass and $M$ is the mass of the black hole. The upper limit is due to gravitational radiation, which is important for heavy strings, while the lower limit corresponds to friction due to the surrounding matter, which is important for light strings. In the next section we will be interested in $\mathcal{P}_{\mathrm{BH}}$ for black holes of mass $M \sim 10^{19} m_{P}$, so Eq. (12) leaves us with a wide range of values of $G \mu$ for which (11) is valid.

\section{OBSERVATIONAL CONSTRAINTS ON LOOP NUCLEATION}

During inflation, loops of size $\sim H^{-1}$ are produced at a constant rate per unit physical volume. After nucleation these loops grow like the scale factor, so we expect that the number of loops with size $\sim R$ contained in a volume $\sim R^{3}$ will be independent of $R$. That is, we expect a scale-invariant distribution of loops. The number density distribution of loops will be given by [1]

$$
\frac{d N}{d V}=\nu \frac{d R}{R^{4}}
$$

Actually, the distribution (13) has a lower cutoff at $R \sim$ $H^{-1}$, since we do not have loops smaller than that. It also has an upper cutoff at $R \sim E H^{-1}$, where $E$ is the total $e$-folding factor since the onset of inflation. See Ref. [1] for details.]

The coefficient $\nu$ is the number of loops produced during an expansion time $H^{-1}$ in a volume $H^{-3}$. This has been estimated in the semiclassical approximation in Ref. [1], using the instanton methods [3]:

$$
\nu=A e^{-B} \text {. }
$$

Here, as in Sec. II, $B=4 \pi \mu / H^{2}$ is the Euclidean action of the instanton describing the nucleation of the string (which is just a spherical world sheet of radius $H^{-1}$ ). The coefficient $A$ has not been calculated and we shall leave it as a free parameter here, presumably of order 1.

We should mention that the semiclassical approximation that leads to Eq. (14) is only valid when the Euclidean action is large $B \gg 1$ (that is, the symmetry-breaking scale of the strings, $\mu^{1 / 2}$, should satisfy $\left.\mu^{1 / 2} \gtrsim H\right)$. On the other hand, we do not want $B$ to be too large, since then the density of strings would be unobservable. As we shall see, the most interesting range occurs for $1<B \lesssim 50$.

After inflation, the distribution of loops with $R \gg t$ is still given by (13), since these loops continue to be stretched by the expansion. Loops with a size smaller than the cosmological horizon, $R \ll t$, are not stretched and are simply diluted by the expansion:

$$
\frac{d N}{d V}=\nu\left[\frac{a(R)}{a(t)}\right]^{3} \frac{d R}{R^{4}},
$$

where $a$ is the cosmological scale factor

The distribution of loops (15) is similar to the distribution of loops that would chop off a network of strings that has been produced at a phase transition [27]. In that case, assuming that $\beta$ loops of length $L=\hat{\alpha} t$ are created at time $t$ per horizon volume and expansion time, the resulting distribution would be $d N / d V=$ $\hat{\alpha}^{3} \beta[a(L) / a(t)]^{3} d L / L^{4}$. To make a correspondence with (15) we should take $L \approx 2 \pi R, \hat{\alpha} \approx 2 \pi$, and $\beta \approx \nu$. Despite the similarities between both scenarios, there are also important differences. In the phase transition scenario, loops are chopped off infinite strings at relativistic speeds, and at any time $t$ there are no loops of length greater than $\hat{\alpha} t$. Numerical simulations suggest a very small value of $\hat{\alpha} \lesssim 10^{-3}[13,14]$. On the other hand, in our case there are no infinite strings, loops have zero velocities, and their size distribution (15) extends all the way to $R \sim t$.

Let us now consider various observational constraints that one can place on the parameters of the model.

(a) Constraint from primordial black hole abundance. For loops that enter the horizon during the radiationdominated era, we have

$$
\frac{d N}{d V}=\nu\left(\frac{1}{t R}\right)^{3 / 2} \frac{d R}{R}
$$

Multiplying by the mass of the loops, $M \approx 2 \pi \mu R$, we obtain the mass density distribution

$$
d \rho(M)=\nu\left(\frac{2 \pi G \mu}{t G M}\right)^{3 / 2} d M
$$

Dividing by the critical density $\rho_{c}=3 / 32 \pi G t^{2}$ we have, at the present time,

$d \Omega_{s}(M) \equiv \frac{d \rho(M)}{\rho_{c}} \approx \frac{64 \pi^{2} \sqrt{2 \pi}}{3}(G \mu)^{3 / 2} \nu\left(\frac{t_{\mathrm{eq}}}{G M}\right)^{1 / 2} \frac{d M}{M}$,

where $t_{\text {eq }} \approx 4 \times 10^{10} \mathrm{sec}$ is the time of equal matter and radiation densities.

As we described in Sec. II, some of the loops in the network will form black holes with probability given by Eq. (11). To obtain the spectrum of primordial black holes that are produced through this mechanism we multipy (18) by $\mathcal{P}_{\mathrm{BH}}$. The fraction of the density parameter $\Omega$ in black holes of mass $\sim M$ is then given by

$\Omega_{\mathrm{BH}}(M) \approx \mathcal{P}_{\mathrm{BH}} \frac{64 \pi^{2} \sqrt{2 \pi}}{3}(G \mu)^{3 / 2} \nu\left(\frac{t_{\mathrm{eq}}}{G M}\right)^{1 / 2}$.

The strongest observational constraint on the abundance of primordial black holes comes from the emission of $\gamma$ rays by holes that are evaporating at the present time [15] (see also [16]). These black holes have a mass $M \approx 5 \times 10^{14} \mathrm{~g}$, and the constraint is given by

$$
\Omega_{\mathrm{BH}}\left(5 \times 10^{14} \mathrm{~g}\right) \lesssim_{10^{-8}} .
$$


From (19) we have

$$
\mathcal{P}_{\mathrm{BH}}(G \mu)^{3 / 2} \nu \lesssim 10^{-28}
$$

We can rewrite this inequality as

$$
-\log _{10} \mathcal{P}_{\mathrm{BH}}\left(L_{*}\right)-\frac{3}{2} \log _{10}(\alpha G \mu)+\left(\log _{10} e\right) B \gtrsim N
$$

where $N=28+\log _{10}\left(A \alpha^{-3 / 2}\right)$. Note that the lefthand side only depends on $B$ and the combination $\alpha G \mu$. Therefore, for given $N$, Eq. (22) will exclude a certain region in the plane $(B, \alpha G \mu)$. Since the parameters $\alpha$ and $A$ are expected to be of order 1 , their contribution to $N$ will be small. Note also that even if the observational constraint (20) was improved by 1 order of magnitude, this would only increase $N$ by one. At any rate, we expect $N \approx 28$ plus or minus a few units.

In Fig. 2, the boundary of the excluded region in parameter space is depicted for $N=27,28$, and 30 . It is seen that the result is not very sensitive to the value of $N$ and, quite generically, the bound will be satisfied provided that

$$
\alpha G \mu \lesssim 10^{-4},
$$

independently of $B$. This can be easily understood since, for such values of $\alpha G \mu$, the parameter $L_{*}$ will be very large and, correspondingly, the probability of black hole formation will be exponentially small (see Fig. 1).

Also, from Fig. 2 we see that for large values of $B$, say $B \gtrsim 50$, the constraint on $G \mu$ is practically removed due to the exponential suppression in the number of nucleated loops. Of course this limit is not very interesting cosmologically.

The bound (23) does not apply for global strings, which form as a result of global symmetry breaking. Fort and

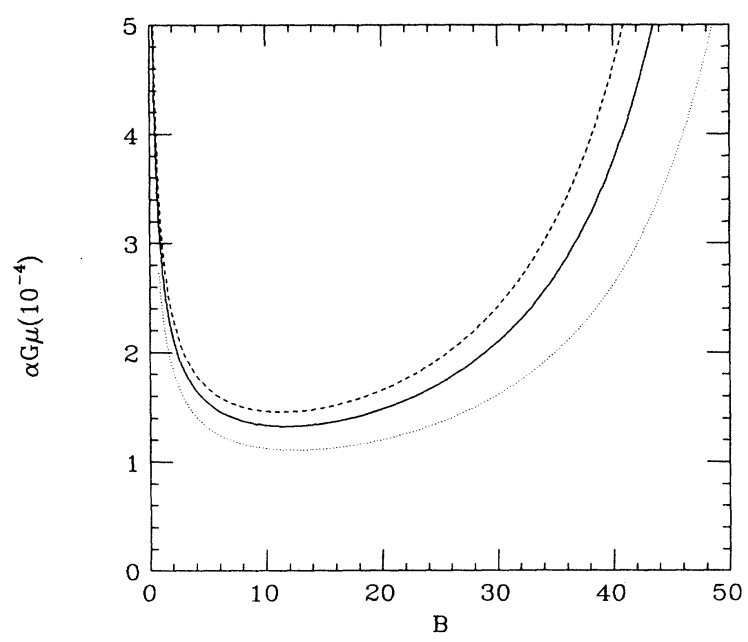

FIG. 2. We represent the boundary of the region excluded by the constraint (22) in parameter space ( $\alpha G \mu$ vs $B)$ for $N=27$ (dashed line), $N=28$ (solid line), and $N=30$ (dotted line). The result is not very sensitive to the value of $N$ and, quite generically, the bound is satisfied provided that $\alpha G \mu \lesssim 10^{-4}$.
Vachaspati [17] have shown that even an exactly circular loop of global string will not form a black hole unless $G \mu \gtrsim 10^{-2}$. The reason is that, unlike gauge strings, global strings emit all of their energy in the form of Goldstone bosons before they reach the Schwarzschild radius. In the following discussion [constraints (b)] we shall see that the gauge string constraint (23) is also in no danger of being violated.

(b) Constraints on the scale of inflation. The background of gravitational waves that would be produced during inflation [18] can be used to constrain the scale of inflation to be less than [19]

$$
H<4 \times 10^{-5} m_{P}
$$

(In certain models of inflation this bound can be tightened by a factor of about 2 [20], due to the contribution of scalar modes to the distortions of the cosmic microwave background.)

From this we have $B>4 \pi \times 10^{9} G \mu$, and if we require $B<50$ we must have

$$
G \mu \lesssim 10^{-8} \quad \text { (gauge strings) }
$$

and thus the black hole constraint (23) is always satisfied.

Global strings produced during inflation can be slightly heavier than that if their thickness is close to $H^{-1}$. In this case [2] $B \approx\left(8 \pi^{2} / 3\right) \lambda \eta^{4} H^{-4}$, where $\lambda$ is a coupling constant not much smaller than 1 and $\eta$ is the vacuum expectation value of the field in the broken phase. For $B \lesssim 50$ we have $G \eta^{2} \lesssim 10^{-9}$. The actual energy per unit length of the string is given by $\mu=2 \pi \eta^{2} \ln (R / \delta)$, where $R$ is the radius of the loop and $\delta=\lambda^{-1 / 2} \eta^{-1}$ is the thickness of the string core. Since the logarithmic factor can be as large as $10^{2}$, we can have $G \mu$ as large as

$$
G \mu \lesssim 10^{-6} \quad \text { (global string) }
$$

and in this case also the black hole constraint (23) is satisfied.

(c) Constraint from gravitational radiation. The millisecond pulsar observations place a constraint on the density parameter in gravitational radiation of a period $\sim 1$ y [21]:

$$
\Omega_{g}<4 \times 10^{-7} h^{-2} .
$$

From strings, we have [22] (see also [23])

$$
\Omega_{g} \sim \frac{128 \pi}{9} \nu\left(\frac{G \mu}{\gamma_{g}}\right)^{1 / 2} \Omega_{r}
$$

where $\Omega_{r} \sim 4 \times 10^{-5} h^{-2}$ is the present density parameter in radiation and $\gamma_{g} \sim 100$. With this, the bound (26) reads

$$
\nu(G \mu)^{1 / 2} \lesssim 2 \times 10^{-3}
$$

This is always satisfied provided that the bounds (24) and (25) from the previous subsection are satisfied.

(d) Structure formation. A question of cosmological interest is whether the constraints derived so far are compatible with a scenario in which the nucleated strings 
would seed the formation of large-scale structure in the Universe. Let us make a rough estimate of the values of the parameters needed for such a purpose.

Assuming, for simplicity, cold dark matter, the mass accreted by a loop of radius $R<t_{\text {eq }}$ at the present time is

$$
M=2 \pi \mu R z_{\text {eq }} \quad\left(R<t_{e q}\right) .
$$

Here $z_{\mathrm{eq}} \approx 2 \times 10^{-4} h^{2}$ is the redshift at time $t_{\mathrm{eq}}$, with $h$ the Hubble constant in units of $100 \mathrm{~km} \mathrm{sec}^{-1} \mathrm{Mpc}^{-1}$. This is because perturbations start growing at time $t_{\text {eq }}$ and they grow proportionally to the scale factor. From (16) the number density of loops of radius $\sim R$ is $n_{R} \sim \nu\left(t_{\mathrm{eq}} R\right)^{-3 / 2} z_{\mathrm{eq}}^{-3}$. Using (27), the mean separation between objects of mass $M$ is given by

$$
d_{M} \sim n_{R(M)}^{-1 / 3} \sim \nu^{-1 / 3}\left(\frac{M t_{\mathrm{eq}} z_{\mathrm{eq}}}{2 \pi \mu}\right)^{1 / 2} .
$$

Rich clusters of mass $M_{\mathrm{cl}} \sim 10^{15} h^{-1} M_{\odot}$ are separated by distances of order $d_{\mathrm{cl}} \sim 50 h^{-1} \mathrm{Mpc}$. Therefore, from (28), we obtain the normalization

$$
\nu^{2 / 3} G \mu \approx 5 \times 10^{-8} h^{-1} \quad\left(\nu \lesssim 10^{-2}\right) .
$$

This normalization is valid for $G \mu \gtrsim 10^{-6} h$ (hence $\nu \lesssim 10^{-2}$ ). For smaller values of $G \mu$ the loops that accrete masses of order $M_{\mathrm{cl}}$ have $R>t_{\mathrm{eq}}$, and (27) does not apply.

For $G \mu \lesssim 10^{-6} h$, loops start accreting matter when they enter the horizon, and we have

$$
M=2 \pi \mu \nu R\left(\frac{t_{0}}{R}\right)^{2 / 3} \quad\left(R>t_{\mathrm{eq}}\right) .
$$

Using $n_{R}=\nu /\left(R t_{0}^{2}\right)$ we obtain

$$
d_{M} \sim n_{R(M)}^{-1 / 3} \sim \nu^{-1 / 3} M(2 \pi \mu)^{-1} .
$$

Again, normalizing for rich clusters, we find

$$
\nu^{1 / 3} G \mu \approx 2 \times 10^{-7} \quad\left(\nu \gtrsim 10^{-2}\right) .
$$

As shown in the discussion of constraints (b), gauge strings can nucleate in substantial numbers only if $G \mu \lesssim 10^{-8}$, and therefore they will not be useful as seeds for structure formation. For global strings the situation is somewhat better since then $G \mu$ can be as large as $\sim 10^{-6}$, so a scenario in which nucleated strings may seed some of the observed structure in our Universe does not seem to be ruled out.

We should now mention the effects of compensation [24] which has been ignored in the above discussion. When a loop nucleates, its energy is balanced by a corresponding deficit in the local densities of matter and radiation. This compensation reduces the gravitational effect of the loop on surrounding matter and on background photons on scales greater than $R$. When a compensated loop radiates away its energy, it leaves behind an underdense region. As the density contrast grows, this region will evolve into a void with a dense central object seeded by the loop. The compensation is somewhat reduced by radiation and neutrinos streaming into the underdense regions and can be further reduced by loop fragmentation. The effect of compensation on structure formation has not been fully investigated, but it seems reasonable to assume that this effect will not be dramatic on scales crossing the horizon before $t_{\text {eq }}$. Then the relations (29) and (30) should still be valid by order of magnitude.

\section{DOMAIN WALLS AND MONOPOLES}

Just as in the case of strings, spherical domain walls can spontaneously nucleate at a constant rate per unit volume during inflation. As a result, the Universe will get filled with a scale-invariant distribution of walls given by (13) and (14), where now $B=2 \pi^{2} \sigma H^{-3}$ is the action of the instanton describing the nucleation of the wall.

The observational constraints on domain wall nucleation are very different from the ones that one can impose on strings, since the mass of a spherical wall

$$
M=4 \pi \sigma R^{2}
$$

grows quadratically rather than linearly with the radius. An inmediate consequence is that walls of size

$$
R_{c}>(8 \pi G \sigma)^{-1}
$$

will all collapse to form black holes [1], since for them $R_{S}>R_{c}$.

To estimate the microwave background anisotropy induced by domain wall bubbles, one has to take proper account of the compensation effect discussed at the end of the previous section. This is a somewhat complicated issue and we shall not attempt to address it in detail here. However, one can make a rough estimate of what this effect should be by using the following arguments.

In a pure dust Universe the compensation would be complete, and background photons would be unperturbed on scales greater than the comoving scale of $R_{S}$ at horizon crossing. Therefore, on larger scales, the whole effect should be due to the underdensity in radiation that is needed to compensate for the mass of the black hole. This underdensity causes radiation from neighboring regions to move in, partially destroying compensation. As this happens, the initial underdensity propagates away from the black hole as a sound wave at the speed of light. At time $t_{\text {eq }}$ the mass of the underdensity, $M_{r}$, is comparable to the mass of the black hole, $M_{\mathrm{BH}}$. At later times, we have $M_{r} \approx M_{\mathrm{BH}} z / z_{\text {eq }}$, due to cosmological redshift. The gravitational potential of $M_{r}$ extends up to scales of order $t$, and we have $\phi \sim G M_{r} t^{-1}$. This will cause temperature distortions of order

$$
\frac{\delta T}{T} \sim \phi \sim \frac{G M_{\mathrm{BH}}}{t_{0}} \frac{z^{5 / 2}}{z_{\mathrm{eq}}},
$$

on scales of the order of the cosmological horizon at redshift $z$.

The angle $\alpha$ subtended by the horizon at redshift $z$ is given by $\tan \alpha \approx\left(z^{1 / 2}-1\right)^{-1}$, so at small angles $\delta T / T \propto$ $\alpha^{-5}$. In particular, for $\alpha \approx 10^{\circ}$, we have 


$$
\frac{\delta T}{T} \sim \frac{M_{\mathrm{BH}}}{M_{u}} \quad\left(\alpha \approx 10^{\circ}\right),
$$

whereas for $\alpha \approx 90^{\circ}$ we have a much lower effect $\delta T / T \sim$ $M_{\mathrm{BH}} /\left(M_{u} z_{\text {eq }}\right)$. Here $M_{u} \equiv G^{-1} t_{0}$ is approximately the mass of our observable Universe.

Let us denote by $R_{\max }$ the radius of the largest wall that may have existed in our observable universe (up to distances $d_{0}=3 t_{0}$ ). How massive can this wall be? From (33), and using $\delta T / T \lesssim 10^{-5}$, we have

$$
M_{\max } \lesssim 10^{-5} M_{u} \approx 10^{52} \mathrm{~g} .
$$

From (31) and (34) we can see that $R_{\max }$ must be less than $t_{\text {eq }}$ even if $\sigma$ is as low as the electroweak scale. Using

$$
\frac{d N}{d V}=\frac{\nu}{R^{5 / 2} t_{\mathrm{eq}}^{3 / 2}}\left(\frac{t_{\mathrm{eq}}}{t_{0}}\right)^{2} d R,
$$

the number of walls with size larger than $R$ within a volume $4 \pi / 3\left(3 t_{0}\right)^{3}$ is

$$
N_{>R}=24 \pi \nu \frac{t_{\mathrm{eq}}^{1 / 2} t_{0}}{R^{3 / 2}} .
$$

Therefore, the larger wall that we can expect to find in the observable Universe has radius $R_{\max } \approx$ $\left[24 \pi \nu t_{\mathrm{eq}}^{1 / 2} t_{0}\right]^{2 / 3}$, and Eq. (34) yields the bound

$$
\nu^{4 / 3} \frac{\sigma}{m_{P}^{3}} \lesssim 10^{-64}
$$

Let us now turn to monopole pair production during inflation. Unlike walls and strings, monopoles are not stretched to enormous sizes by the inflationary expansion. They are just diluted. An inmediate consequence is that the only monopoles that will be relevant at the end of inflation are the ones that have been produced during the last expansion time. This density is given by [1]

$$
n=H^{3} A e^{-2 \pi m / H},
$$

where $m$ is the mass of the monopole (here, as in the previous sections, the exponent $2 \pi m H^{-1}$ is the action of the relevant instanton).

A constraint on the abundance of nucleated monopoles comes from the fact that they should not recreate the monopole problem that inflation was aimed to solve. Of course this can be easily achieved by choosing the ratio $m / H$ to be sufficiently large, but as we shall see, one does not necessarily have to impose that.

Actually, the number density of monopoles at the time of reheating depends very much on the details of how inflation ended. Consider, as an example, a model in which the usual exponential expansion is followed by a short period of power law expansion $a \propto t^{p}$ that starts at time $t_{1}$ and ends at time $t_{2}$ ( $p$ may be larger than 1 , in which case we have power law inflation, but it need not be). At time $t_{2}$ reheating is completed and we enter the usual radiation-dominated era.

It is clear that, if no monopoles are created during the power law epoch, we will have, at time $t_{2}$,

$$
n\left(t_{2}\right)=n\left(t_{1}\right) f^{3 p},
$$

where $n\left(t_{1}\right)$ is given by (36) and

$$
f \equiv\left(\frac{t_{1}}{t_{2}}\right) \approx 1.6 \mathcal{N}^{1 / 2} \frac{T_{r}^{2}}{H m_{P}} .
$$

Here $\mathcal{N}$ is the effective number of massless degrees of freedom at reheating and we have used $t_{1}=p H^{-1}$ and $t_{2}=2 p m_{P}(10 \mathcal{N})^{-1 / 2} T_{r}^{-2}$, with $m_{P}$ the Planck mass and $T_{r}$ the reheating temperature. Even if we assume that monopoles continue to be produced at a rate per unit volume given by

$$
A \mathcal{H}^{4} e^{-\frac{2 \pi m}{\mathcal{H}}}
$$

where $\mathcal{H} \equiv \dot{a} / a$ is the instantaneous expansion rate, it is not difficult to show (for $2 \pi m H^{-1}>3 p-4$ ) that the density of monopoles during the power law era is dominated by the ones that were already present at $t_{1}$. Therefore Eq. (37) is still valid in this case.

After reheating, the monopoles continue to be diluted as $a^{-3}$ whereas the density in radiation decays as $a^{-4}$. Therefore the contribution of the monopoles to the density parameter at the time $t_{\mathrm{eq}} \approx 10^{10} \mathrm{sec}$ given by

$$
\begin{aligned}
\Omega_{m} & =\frac{m n\left(t_{2}\right)}{\rho_{c}\left(t_{2}\right)}\left(\frac{t_{\mathrm{eq}}}{t_{2}}\right)^{1 / 2} \\
& \approx 10^{29} f^{3\left[p-\frac{1}{2}\right]}\left[\frac{H}{m_{P}}\right]^{3 / 2} A e^{-\frac{2 \pi m}{H}} \frac{m}{m_{P}} .
\end{aligned}
$$

The Parker bound [25] requires $\Omega_{m} \lesssim 10^{-4}\left(\mathrm{~m} / \mathrm{m}_{P}\right)$ (assuming Dirac charges for the monopoles), so we must impose

$$
\left[\frac{H}{m_{P}}\right]^{3 / 2} f^{3\left[p-\frac{1}{2}\right]} A e^{-\frac{2 \pi m}{H}} \lesssim 10^{-33} .
$$

As mentioned above, this bound can be trivially satisfied by supressing the creation of monopoles with a sufficiently large $m / H$ ratio. Alternatively, if $p>1 / 2$, then $\Omega_{m}$ will decrease with the reheating temperature through the supression factor $f$. For instance, taking $p=3.5$ and $f \sim 10^{-4}$ the bound is automatically satisfied without any assumptions on $m / H$.

\section{CONCLUSIONS}

In this paper we calculated the probability of black hole formation by a string loop spontaneously nucleated during inflation. The calculation was based on the quantum theory of perturbations on strings in de Sitter space developed in Ref. [8]. The result is given in Eq. (11) and in Fig. 1. We then used probability (11) to derive observational constraints on the mass parameter of the string and on the loop density parameter $\nu$. The strongest constraint comes from the emission of $\gamma$ rays by evaporating black holes. The excluded region in the parameter space is shown in Fig. 2. Roughly, the values of the parameters for which both $G \mu \gtrsim 10^{-4}$ and $\mu \lesssim 4 H^{2}$ are excluded. 
This bound does not apply to global strings which, for $G \mu \lesssim 10^{-2}$, radiate their energy into Goldstone bosons before they reach the Schwarzschild radius [17]. In the interesting case when the string thickness is comparable to $H^{-1}$, the expression for the Euclidean action is also modified and the excluded parameter space for global strings is $G \mu \gtrsim 10^{-2}$ with $\eta \sim H$, where $\eta$ is the vacuum expectation value (VEV) of the field in the broken phase.

The allowed rate of expansion during inflation, $H$, is limited $[18,19]$ by the observational bounds on the intensity of long-wavelength gravitational waves, $H<$ $4 \times 10^{-5} m_{P}$. This bound implies that loops of string can nucleate in substantial numbers only if $G \mu \lesssim 10^{-8}$, for gauge strings, or $G \mu \lesssim 10^{-6}$ for global strings. In both cases, this bound is much stronger than the corresponding black hole constraint, and thus black holes can only form with a density well below the present observational constraints. Gauge strings with $G \mu \lesssim 10^{-8}$ are too light to serve as seeds for structure formation, while global strings may still play this role if $G \mu$ is near its upper bound, $G \mu \sim 10^{-6}$.

We have also briefly discussed the cosmological implications of nucleated domain wall bubbles and monopoleantimonopole pairs. For sufficiently large bubbles, the formation of black holes is inevitable. Although these black holes can have enormous masses, their effect on structure formation and on the background radiation anisotropy is diminished due to the compensation effect [24]. The predicted density of the monopoles is given by Eq. (38). This density is not in conflict with the Parker bound on the cosmological abundance of monopoles provided that the parameters lie in the range determined by Eq. (39).

\section{ACKNOWLEDGMENTS}

This research was supported in part by the National Science Foundation under Grant Nos. PHY 89-04035 and PHY 87-14654, and J. G. was supported by a Fulbright grant. A. V. is grateful to Sidney Coleman and to the High Energy Theory group at Harvard for their hospitality.

\section{APPENDIX A}

In this appendix we study linear perturbations to a circular loop of string in flat space. Before going to the particular case of a circular loop, we shall consider perturbations to an arbitrary string configuration whose world sheet is given by $x^{\mu}\left(\xi^{a}\right)$ ( $\xi^{a}$ are arbitrary coordinates on the world sheet). This will be a trivial extension of the general results given in Refs. $[7,8]$ for the case of domain walls.

Following Ref. [7], we parametrize the perturbed world sheet $\tilde{x}^{\mu}$ as

$$
\tilde{x}^{\mu}(\xi)=x^{\mu}(\xi)+\sum_{A=1}^{2} n^{A \mu} \phi^{A},
$$

where $n^{A \mu}$ are the two vectors normal to the unperturbed world sheet. They satisfy

$$
n^{A \mu} \partial_{a} x_{\mu}=0, \quad n^{A \mu} n_{\mu}^{B}=\delta^{A B} .
$$

The perturbation fields $\phi^{A}$ have the meaning of normal displacements to the world sheet, as measured by an observer that is moving with the unperturbed string.

The dynamics of the string is governed by the Nambu action

$$
S\left[x^{\mu}(\xi)\right]=-\mu \int \sqrt{-g} d^{2} \xi
$$

where $g$ is the determinant of the metric induced on the world sheet $g_{a b}=\partial_{a} x^{\mu} \partial_{b} x_{\mu}$. The equations of motion that result from (A3) are well known:

$$
\square x^{\mu}\left(\xi^{a}\right)=0,
$$

where $\square$ stands for the covariant d'Alembertian on the world sheet. Multiplying (A4) by $n_{\mu}^{A}$ and integrating by parts one obtains

$$
g^{a b} K_{a b}^{A}=0
$$

where $K_{a b}^{A} \equiv-\partial_{a} n_{\mu}^{A} \partial_{b} x^{\mu}$ is the extrinsic curvature corresponding to the normal $n^{A}$.

The effective action for the perturbation fields $\phi^{A}$ on a given background $x^{\mu}(\xi)$ can be obtained by introducing (A1) into the action (A3) and then expanding to quadratic order in $\phi^{a}$. After some lengthy algebra, the result can be written as

$$
S\left[\tilde{x}^{\mu}\right]=S\left[x^{\mu}\right]+S_{\phi}
$$

The first term is just the action for the unperturbed solution, while the second is given by

$S_{\phi}=-\mu \int \sqrt{-g}\left[\frac{1}{2} \phi_{, a}^{A} \phi^{A},{ }^{a}-\frac{1}{2} K_{a b}^{A} K^{B a b} \phi^{A} \phi^{B}+\mathcal{S}\right] d^{2} \xi$,

with

$$
\mathcal{S}=\frac{1}{2} \phi^{A} \phi^{B} n^{C \mu} n^{C \nu} \partial_{a} n_{\mu}^{A} \partial^{a} n_{\nu}^{B}+\phi_{, a}^{A} \phi^{B} n^{A \mu} n_{\mu}^{B},{ }^{a} .
$$

In deriving this effective action we have used the equations of motion (A5) to eliminate the terms linear in $\phi^{A}$. From (A6) we see that, in general, the two fields $\phi^{A}$ are coupled to each other in a complicated way.

Particularizing to the case of a circular loop, matters will simplify considerably. The unperturbed world sheet in Cartesian coordinates $(t, x, y, z)$ is given by

$$
x^{\mu}=\left(t, R_{c} \cos \theta \cos \frac{t}{R_{c}}, R_{c} \sin \theta \cos \frac{t}{R_{c}}, 0\right) .
$$

Here $R_{c}$ is just the radius of the loop at $t=0$ (when the loop is at rest). The two vectors normal to the world sheet, can be chosen as 


$$
\begin{aligned}
& n_{\mu}^{(1)}=\frac{1}{\cos \frac{t}{R_{c}}}\left(\sin \frac{t}{R_{c}}, \cos \theta, \sin \theta, 0\right), \\
& n_{\mu}^{(2)}=(0,0,0,1) .
\end{aligned}
$$

The first one is a radial normal vector, while the second one is transverse to the plane of the loop. It is easy to see that in this case $K_{a b}^{(2)}=0$ and $\mathcal{S}=0$, so the fields $\phi^{(1)}$ and $\phi^{(2)}$ decouple from each other. They simply behave like free scalar fields in the curved geometry of the unperturbed world sheet.

In particular, the transverse perturbation behaves like a massless minimally coupled field. The corresponding equation of motion

$$
\square \phi^{(2)}=\frac{1}{\sqrt{-g}} \partial_{a}\left(\sqrt{-g} g^{a b} \partial_{b} \phi^{(2)}\right)=0
$$

can be solved trivially. The metric on the world sheet is given by

$$
d s_{\Sigma}^{2}=g_{a b} d \xi^{a} d \xi^{b}=\cos ^{2}\left(t / R_{c}\right)\left[-d t^{2}+R_{c}^{2} d \theta^{2}\right]
$$

Introducing this metric in (A9) we obtain a flat space wave equation

$$
\ddot{\phi}^{(2)}-\frac{1}{R_{c}^{2}} \phi^{\prime \prime(2)}=0,
$$

where a prime denotes derivative with respect to $\theta$. Equation (A10) is not surprising, since in $1+1$ dimensions the conformal coupling is the same as the minimal coupling (see, e.g., [26]). The mode solutions of (A10) are just standing waves that oscillate with constant amplitude. Note that $\phi^{(2)}$ coincides with $\Delta_{r}$ of Sec. II, so we have the following result: transverse perturbations to a circular loop oscillate with constant amplitude as the loop collapses.

The equation of motion for the radial perturbations $\phi^{(1)}$ can be found from (A6) (with $K_{a b}^{(2)}=\mathcal{S}=0$ ). We have

$$
\square \phi^{(1)}+K_{a b}^{(1)} K^{(1) a b} \phi^{(1)}=0 .
$$

The extrinsic curvature can be obtained from (A7) and (A8):

$$
K_{t t}^{(1)}=\frac{1}{R_{0}}, \quad K_{\theta \theta}^{(1)}=R_{0}, \quad K_{t \theta}^{(1)}=0 .
$$

The proper perturbation $\phi^{(1)}$, as measured by a local observer that is moving with the string, is related to the radial perturbation $\Delta_{r}$ defined in Sec. II through a Lorentz contraction factor

$$
\Delta_{r}=\phi^{(1)} \sqrt{1-\dot{R}^{2}}
$$

Writing (A11) in terms of $\Delta_{r}$ and decomposing in Fourier modes [as in (4)] we have, after some algebra,

$$
\ddot{\Delta}_{r, L}+\frac{2}{R_{c}} \tan \left(\frac{t}{R_{c}}\right) \dot{\Delta}_{r, L}+\frac{\left(L^{2}-1\right)}{R_{c}^{2}} \Delta_{r, L}=0 .
$$

The general solution to this equation can be written as

$$
\begin{aligned}
\Delta_{L}(t)=\frac{\Delta_{L}(0)}{L}\left[\sqrt{1-\frac{R^{2}}{R_{c}^{2}}} \sin \frac{L t}{R_{c}}+L \frac{R}{R_{c}} \cos \frac{L t}{R_{c}}\right] \\
+\dot{\Delta}_{L}(0) \frac{R_{c}}{1-L^{2}}\left[\sqrt{1-\frac{R^{2}}{R_{c}^{2}}} \cos \frac{L t}{R_{c}}\right. \\
\left.-L \frac{R}{R_{c}} \sin \frac{L t}{R_{c}}\right],
\end{aligned}
$$

where $\Delta_{L}(0)$ and $\dot{\Delta}_{L}(0)$ are the values of the perturbation and its derivative at $t=0$ (we have dropped the subindex $r)$, and $R=R_{c} \cos \left(t / R_{c}\right)$ is the radius of the unperturbed loop.

In the cosmological problem that we are interested in, $t=0$ corresponds to the moment when the loop enters the horizon and starts collapsing. The probability distribution for the initial conditions $\Delta_{L}(0)$ will be given by (5) and (7). We also need the initial conditions $\dot{\Delta}_{L}(0)$. When the wavelength of a perturbation is within the horizon we expect $\left\langle\dot{\Delta}_{L}^{2}\right\rangle=\left(L / R_{c}\right)^{2}\left\langle\Delta_{L}^{2}\right\rangle$, where the angular brackets indicate the average over one oscillation period. Therefore, by the time the loop enters the horizon, the perturbations will have developed velocities of order $\dot{\Delta}(t=0) \sim\left(L / R_{c}\right) \Delta_{L}(t=0)$. Using this in (A12) we have

$$
\Delta_{L}\left(R \ll R_{c}\right) \approx \frac{\Delta_{L}\left(R_{c}\right)}{L} .
$$

That is to say, the radial perturbations shrink by a factor of $L$ as the loop collapses.

\section{APPENDIX B}

Damping mechanisms, such as gravitational radiation and friction, may decrease the amplitude of the perturbations on a circular loop of string and therefore increase the probability of black hole formation. In this appendix we briefly discuss the limits in which the effects of gravitational radiation and friction can be ignored.

Gravitational radiation can only smooth out perturbations on wavelengths smaller than $\lambda_{g} \equiv \gamma_{g} G \mu t$, where $\gamma_{g} \sim 100$ (see, e.g., [27]). Taking $t \sim R_{c}$, this corresponds to wave numbers larger than $L_{g} \equiv 2 \pi\left(\gamma_{g} G \mu\right)^{-1}$. It is clear, from the physical interpretation of $L_{*}$ discussed in Sec. II, that gravitational damping can be neglected in the calculation of $\mathcal{P}_{\mathrm{BH}}$ providing that $L_{g}>L_{*}$. This inequality gives

$G \mu<\left(2 \pi \gamma_{g}^{-1}\right)^{5 / 3}\left(16 \pi^{2} \alpha^{2} B\right)^{1 / 3} \approx\left(17 \gamma_{g}^{-1}\right)^{5 / 3}\left(\alpha^{2} B\right)^{1 / 3}$.

Typically, the right-hand side of (B1) will be of order $10^{-2}$ or larger, so this is not a very strong condition.

Similarly we can consider the effects of friction. The dominant contribution to friction comes from AharonovBohm scattering of ambient particles off the string in the radiation dominated era (see Ref. [28]). Assuming a situation in which the wavelength of the perturbations is 
conformally stretched by the expansion of the Universe (as it is in the present case), it is shown in Ref. [29] that friction can only be important for wavelengths smaller than $\lambda_{f} \equiv \gamma_{f}(G \mu T)^{-1}$. Here $T$ is the temperature and $\gamma_{f}$ is a numerical coefficient of order 1 . The physical reason is that for $\lambda>\lambda_{f}$, the friction term in the equations of motion will "switch off" before the perturbations cross the cosmological horizon and start to oscillate.

The wave number corresponding to $\lambda_{f}$ is

$$
L_{f} \equiv 2 \pi \gamma_{f}^{-1} R T G \mu=\gamma_{f}^{-1} G M T_{c},
$$

where $T_{c}$ is the temperature of the Universe at the time $t_{c}$ when the loop crosses the horizon, $M$ is the mass of the loop at $t_{c}$ (which is also the mass of the resulting black hole), and we have used that the product $R T$ is independent of time before the loop crosses the horizon. Using $T_{c} \approx(10 G \mathcal{N})^{-1 / 4} t_{c}^{-1 / 2}$ and $t_{c} \approx R_{c}=M(2 \pi \mu)^{-1}$ (where $\mathcal{N} \sim 10^{2}$ is the effective number of massless degrees of freedom) we have

$$
L_{f} \equiv(10 \mathcal{N})^{-1 / 4} \gamma_{f}^{-1}(2 \pi G \mu)^{1 / 2}\left(\frac{M}{m_{P}}\right)^{1 / 2} .
$$

Similar to the case of gravitational radiation, friction can be neglected in the calculation of $\mathcal{P}_{\mathrm{BH}}$ providing that $L_{f}>L_{*}$. This condition is equivalent to

$$
G \mu \gtrsim\left(\frac{\mathcal{N} \gamma_{f}^{4}}{230}\right)^{5 / 18}\left(\alpha^{2} B\right)^{-2 / 9}\left(\frac{m_{P}}{M}\right)^{5 / 9} .
$$

Therefore, the range of values of $G \mu$ for which friction can be ignored depends on the mass of the black hole that one wishes to consider.

Putting (B1) and (B2) together and taking $\gamma_{f} \sim 1$, $\gamma_{g} \sim 10^{2}$, we conclude that damping is unimportant for values of $G \mu$ in the range

$$
\left(\alpha^{2} B\right)^{-2 / 9}\left(\frac{m_{P}}{M}\right)^{5 / 9} \lesssim G \mu<5 \times 10^{-2}\left(\alpha^{2} B\right)^{1 / 3} .
$$

[1] R. Basu, A.H. Guth, and A. Vilenkin, Phys. Rev. D 44, 340 (1991).

[2] R. Basu and A. Vilenkin, Phys. Rev. D 46, 2345 (1992).

[3] For a review of vacuum decay, see, e.g., S. Coleman, $A s-$ pects of Symmetry (Cambridge University Press, Cambridge, England, 1985).

[4] A. Vilenkin, Phys. Rev. Lett. 46, 1169 (1981); 46, 1196(E) (1981).

[5] S.W. Hawking, Phys. Lett. B 231, 237 (1989).

[6] A.G. Polnarev and R. Zembowicz, Phys. Rev. D 43, 1106 (1991).

[7] J. Garriga and A. Vilenkin, Phys. Rev. D 44, 1007 (1991).

[8] J. Garriga and A. Vilenkin, Phys. Rev. D 45, 3469 (1992).

[9] N. Turok, Phys. Rev. Lett. 60, 549 (1988).

[10] T. Vachaspati and A. Vilenkin, Phys. Rev. D 43, 340 (1991).

[11] A. Vilenkin, Phys. Rev. D 24, 2082 (1981).

[12] N. Turok and P. Bhattacharjee, Phys. Rev. D 29, 1557 (1984).

[13] D. Bennett and F. Bouchet, Phys. Rev. Lett. 60, 257 (1988); 63, 2776 (1989).

[14] B. Allen and E.P.S. Shellard, Phys. Rev. Lett. 64, 119 (1990).

[15] J. H. MacGibbon and B. J. Carr, Astrophys. J. 371, 447
(1991).

[16] J.D. Barrow, E.J. Copeland, and A.R. Liddle, Phys. Rev. D 46, 645 (1992).

[17] J. Fort and T. Vachaspati, "Do global strings collapse to form black holes?," Tufts report (unpublished).

[18] A.A. Starobinsky, Pis'ma Zh. Eksp. Teor. Fiz. 30, 719 (1979) [JETP Lett. 30, 682 (1979)].

[19] M. White, Phys. Rev. D 46, 4198 (1992).

[20] A.R. Liddle and D.H. Lyth, Phys. Rep. (to be published).

[21] D.R. Stinebring, M.F. Ryba, J.H. Taylor, and R.W. Romani, Phys. Rev. Lett. 65, 285 (1990).

[22] T. Vachaspati and A. Vilenkin, Phys. Rev. D 31, 3052 (1985).

[23] R.R. Caldwell and B. Allen, Phys. Rev. D 45, 3447 (1992).

[24] S. Veeraraghavan and A. Stebbins, Astrophys. J. 365, 37 (1990).

[25] E.N. Parker, Cosmical Magnetic Fields (Oxford University Press, Oxford, 1979).

[26] N.D. Birrell and P.C.W. Davies, Quantum Fields in Curved Space (Cambridge University Press, Cambridge, England, 1982).

[27] See, e.g., A. Vilenkin, Phys. Rep. 121, 263 (1985).

[28] A. Vilenkin, Phys. Rev. D 43, 1061 (1991).

[29] J. Garriga and M. Sakellariadou, "Effects of friction on strings," Tufts University report (unpublished). 